\title{
Does feticide shorten termination duration in second trimester pregnancy terminations?
}

\author{
Aytek Ş1k ${ }^{1}$, Sedat Bilecan², Serkan Kumbasar, Yaşam Kemal Akpak ${ }^{4}$, Yilda Arzu Aba ${ }^{5}$
}

1. Istanbul Aydın University, Department of Obstetrics and Gynaecology, Istanbul, Turkey.

2. Süleymaniye Research and Education Hospital, Department of Obstetrics and Gynaecology, Istanbul, Turkey.

3. Sakarya University School of Medicine, Sakarya Research and Education Hospital, Department of Obstetrics and Gynaecology, Sakarya, Turkey.

4. Ankara Mevki Military Hospital, Department of Obstetrics and Gynaecology, Ankara, Turkey.

5. Bandırma Onyedi Eylül Üniversitesi, Health Science Faculty, Balikesir, Turkey.

\section{Email:}

Bulat Aytek Ş1k: bulataytek@hotmail.com

Sedat Bilecan: drsedatbilecan@hotmail.com

Serkan Kumbasar: doktor1977@hotmail.com

Yasam Kemal Akpak: yasamaster@gmail.com

Yilda Arzu Aba: yildaarzum@hotmail.com

\begin{abstract}
Backround: A retrospective (case-controlled) study was conducted with the aim of identifying the effect of the use of misoprostol on termination time in patients who did and did not undergo feticide procedures in second trimester pregnancy terminations.

Methods: The sampling of the study consisted of 144 pregnant women who were diagnosed as having major fetal anomalies incompatible with life, and were recommended for termination of pregnancy. The investigation showed that feticide procedures were performed for 99 women, and feticide procedures were not performed for 45 women. Misoprostol protocol was administered for 48 hours in the termination period; whether the feticide procedure directly affected the termination duration in patients who did and did not undergo feticide was evaluated.

Results: Abortion/birth was achieved in 103 (71.5\%) women during the first 48 hours. There was no significant difference between the termination duration of the misoprostol protocol among the women who did and did not undergo feticide. There was no significant difference between the termination durations and fetal biometric measurements (BPD, HC) except head diameters $(\mathrm{p}=0.020$ and $\mathrm{p}=0.015)$.
\end{abstract}

Conclusions: The misoprostol protocol is shown to be effective and safe for the termination of pregnancies during the second trimester. Feticide has no affect on the duration of termination.

Keywords: Feticide, second trimester pregnancy, terminations.

DOI: https://dx.doi.org/10.4314/ahs.v19i1.28

Cite as: Şlk. A, Bilecan S, Kumbasar S, Akpak. YK, YA A. Does feticide shorten termination duration in second trimester pregnancy terminations? Afri Health Sci. 2019;19(1). 1544-1553. https:/ / dx.doi.org/ 10.4314/abs.v19i1.28

\section{Introduction}

Fetal abnormality is known to be a major cause of peri-

\section{Corresponding author:}

Yilda Arzu Aba,

Mailing address: yildaarzum@hotmail.com

Phone: +905055670483

Email: yildaarzum@hotmail.com natal mortality. Termination of pregnancy for fetal abnormalities significantly decreases perinatal mortality resulting from birth defects ${ }^{1,2}$. Fetal morphologic anomalies (without chromosomal anomalies) and chromosomal anomalies formed the main grounds for termination of pregnancy in $39 \%$ and $35 \%$ of cases, respectively, in a study by Guillem et al. ${ }^{3}$ and $47 \%$ and $33 \%$, respectively, in another study by Ramalho et al. ${ }^{4}$. Pregnancies may need to be terminated during the second trimester due

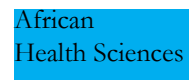

(C) 2019 Ş1k et al. Licensee African Health Sciences. This is an Open Access article distributed under the terms of the Creative commons Attribution License (https://creativecommons.org/licenses/BY/4.0), which permits unrestricted use, distribution, and reproduction in any medium, provided the original work is properly cited. 
to fetal or maternal reasons. Dilation and curettage is the most preferred method for the termination of pregnancies during the first trimester, but termination with this method during the second trimester is likely to cause problems. The width of fetal-placental units, increased uterine blood flow, and the unripe cervix complicate the procedure during the second trimester ${ }^{5}$. Termination during the second trimester is achieved with prostaglandins in today's world. Misoprostol (15-deoxy-16-hydroxy16-methyl prostaglandin E1) is a synthetic Prostaglandin E1 (PGE1) analogue. PGE1 is used in the induction of labor because of its uterotonic effects and it provides cervical ripening ${ }^{6}$.

Due to easy storage at room temperature, cost-effectiveness and availability, misoprostol is the drug of choice. Misoprostol is successfully used in oral, sublingual, intravaginal, intracervical, and rectal forms in the termination of pregnancies ${ }^{7,8}$. However, there is no consensus about which route is the most effective and least likely to cause adverse effects in the termination of second trimester pregnancies. The half life of oral misoprostol is 20-40 minutes, the dose is between $200-800 \mu \mathrm{g}$, and application frequency is between 3-12 hours. Its adverse effects on the gastrointestinal system including nausea, vomiting, fatigue, trembling, and fever are limited ${ }^{6-8}$. If the application route is disregarded, the adverse effects are dependent on the dose.

We aimed to investigate the effect of the use of oral misoprostol after 200 mcg vaginal misoprostol (PGE1-Cytotec) administration on the termination duration in patients who did and did not undergo feticide in second trimester pregnancy terminations.

\section{Materials and methods}

The files of 905 pregnant women who presented to the pre-natal diagnosis unit in Süleymaniye Gynecology and Obstetrics Education and Training Hospital, and were diagnosed as having fetal anomalies in second trimester colour Doppler ultrasonography screening between 2009 and 2014 were retrospectively investigated. 455 out of 905 pregnant women were diagnosed as having major fetal anomalies incompatible with life, and were recommended termination of pregnancy. The ultrasonographic screening of 450 pregnant women revealed minor fetal anomalies compatible with life, and termination was not recommended. The detected major and minor anomalies were central nervous system anomalies, cardiovascular system anomalies, chromosomal anomalies detected in amniocentesis, skeletal system anomalies, and urinary system anomalies.

Two hundred fourteen out 455 pregnant women who were recommended termination accepted to terminate the pregnancy, and 241 pregnant women refused to terminate pregnancy. One hundred forty-four pregnant women out of 214 who accepted termination were included in the study in accordance with the sample inclusion criteria. The inclusion criteria were pregnancies with fetal anomalies that were incompatible with life in the second trimester; being aged over 15 years; Bishop score of 3 or below in bimanual examination; and pregnancies with no placental location anomalies. The exclusion criteria were the pregnancies with major fetal anomalies that were incompatible with life in the first and third trimester; being aged younger than 15 years; having been diagnosed with systemic and chronic diseases such as heart disease, diabetes mellitus, eclampsia-preeclampsia, and asthma; initiated active labor action; Bishop scoring over 3; pregnancies with minor or major fetal anomalies that were compatible with life.

The study was conducted in the perinatology and delivery room unit in Süleymaniye Gynecology and Obstetrics Education and Training Hospital after the approval of the hospital education and planning board and ethics board were granted.

Feticide procedures were performed (case group) for 99 pregnant women out of 144 who were recommended termination. Feticide procedures were not performed (control group) for 45 pregnant women. The feticide procedures were performed by the same physician under transabdominal ultrasonography guidance with a percutaneous injection of potassium chloride (KCL) solution $(1.5 \mathrm{~g} \mathrm{KCL}$ in $10 \mathrm{~mL})$ to the fetal heart or umbilical cord using a 20-gauge spinal needle.

Bimanual examinations of the pregnant women were performed in the delivery room unit. Two hundred micrograms of dry misoprostol (Cytotec ${ }^{\circledR} 200 \mathrm{mcg}$ tablet x 1, Ali Raif Drug Industry Inc., Istanbul, Turkey) was placed into the vaginal posterior fornix. A maximum of 4 doses of oral $400 \mathrm{mcg}$ misoprostol (Cytotec $\AA 200 \mathrm{mcg}$ tablet x, 1 Ali Raif Drug Industry Inc., Istanbul, Turkey) 
was administered every 4 hours to women who had no history of previous cesarean section, and a maximum 8 doses of oral $200 \mathrm{mcg}$ misoprostol were administered every 4 hours to women with a history of previous cesarean section. The women were followed up for 48 hours, after which their condition was evaluated. Misoprostol treatment was accepted as unsuccessful in patients whose pregnancies were not terminated after 48 hours. In unsuccessful cases, additional procedures such as induction with oxytocin (20 drops per minute with 5 IU oxytosin into 500 cc of $5 \%$ dextrose) and placement of a Foley catheter into the uterine cavity were performed (Figure 1).

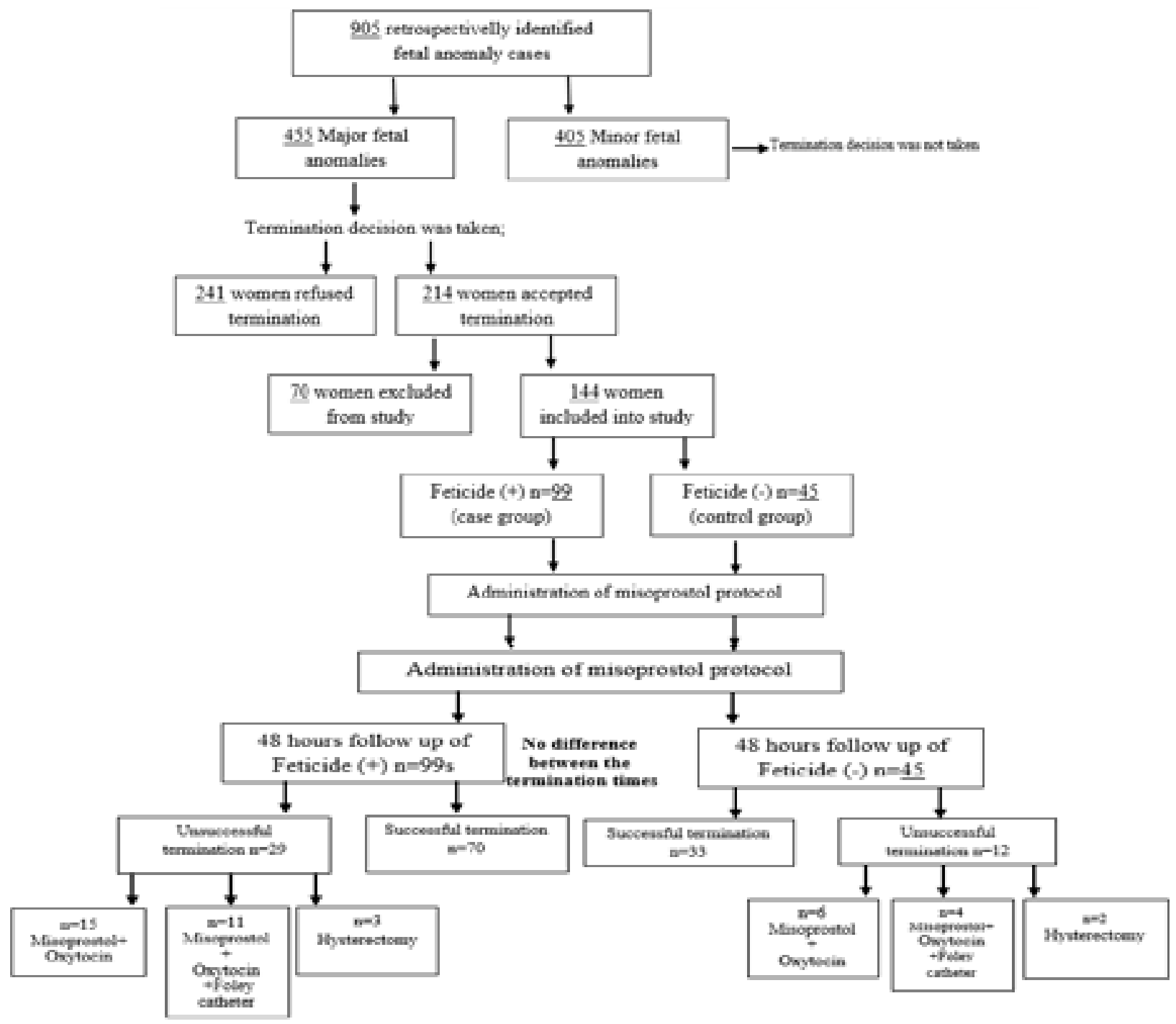

Figure 1. Consort Flow Diagram

The NCSS 2007 and PASS 2008 statistical software (Utah, USA) program was used for statistical analyses for the evaluation of the findings of the study. Pearson's correlation analysis was used for the detection of relationships between the parameters, and the effects of gestational week, biparietal diameter, and head circumference on the termination time were evaluated using linear regression analysis. $\mathrm{P}$ values $<0.05$ were considered significant. 


\section{Results}

The demographic features of the 144 patients are given in Table 1. The mean age of the patients was $27.47 \pm 6.0$ years. The mean gravida was $2.43 \pm 1.56$, the mean parity was $1.76 \pm 1.06$, the mean number of abortions was
$1.48 \pm 0.96$, and the mean gestational week was $21.35 \pm 4.46$. 40 patients were nulliparous, and 104 were multiparous. 44 out of 104 multiparous patients had experienced one or more cesarean deliveries. The mean termination times of the patients was $34.60 \pm 27.31$ hours.

Table 1: Distribution of Demographic Characteristics

\begin{tabular}{lcc}
\hline Demographic Characteristics & Feticide & Feticide \\
& Performed $(\mathbf{n = 9 9 )}$ & Not Performed (n=45) \\
\hline \hline Age (years) (Mean \pm SD) & $26.56 \pm 5.0$ & $28.32 \pm 7.1$ \\
Gravida (Mean \pm SD) & $2.33 \pm 1.61$ & $2.63 \pm 1.09$ \\
Parity (Mean \pm SD) & $1.75 \pm 0.92$ & $1.77 \pm 1.1$ \\
Number of Abortions (Mean \pm SD) & $1.50 \pm 0.99$ & $1.52 \pm 0.82$ \\
Nulliparous (n/percent) & $30(30.3 \%)$ & $10(22.2 \%)$ \\
Multiparous (n/percent) & $69(69.7 \%)$ & $35(77.7$ \\
Previous cesarean-section (n/percent) & $32(32.3 \%)$ & $12(26.7 \%)$
\end{tabular}

The distribution of the fetal anomalies of the $144 \mathrm{pa}-$

tients who were diagnosed as having major fetal anomalies incompatible with life is given in Table 2.

Table 2: Distributions according to anomaly type

\begin{tabular}{lccc}
\hline & Feticide & Feticide & Total \\
& Performed (n=99) & Not Performed (n=45) & (N=144) \\
Anomaly types & $\mathbf{n ~ ( \% )}$ & $\mathbf{n ~ ( \% )}$ & $\mathbf{n ~ ( \% )}$ \\
\hline \hline CNS & $25(25.3)$ & $11(24.5)$ & $36(25)$ \\
Multiple & $24(24.2)$ & $9(20.0)$ & $33(22.9)$ \\
CVS & $19(19.2)$ & $10(22.2)$ & $29(20.1)$ \\
Chromosomal & $9(9.1)$ & $4(8.9)$ & $13(9.1)$ \\
Skeletal system & $7(7.1)$ & $4(8.9)$ & $11(7.6)$ \\
Urinary system & $5(5.1)$ & $2(4.4)$ & $7(4.9)$ \\
GIS & $2(2.0)$ & - & $2(1.4)$ \\
Other & $8(8.1)$ & $5(11.1)$ & $13(9)$ \\
Total & $99(100)$ & $45(100)$ & $144(100)$ \\
\hline
\end{tabular}

Among the 144 patients, the number of women with a history of cesarean sections was 44 . The minimum and maximum values of abortion/birth intervals in patients with a history of cesarean section were 5 hours and 106 hours, respectively; with a mean of $32 \pm 30$ hours and a median of 18 hours. The minimum and maximum values of abortion/birth intervals in multiparous women with no history of cesarean section were 2 hours and 94 hours respectively; with a mean of $23.6 \pm 22.5$ hours and a median of 14.5 hours. The minimum and maximum values of abortion/birth intervals in nulliparous patients without previous cesarean section were 7 hours and 126 hours, respectively; with a mean of $32.9 \pm 24.2$ hours and a median of 27 hours. The time from the initiation of medicine until abortion in nulliparous patients was statistically significantly longer compared with multiparous patients $(p<0.05)$. The dispersion according to abortion/ birth intervals is presented in Table 3 . 
Table 3: Dispersion according to abortion/birth intervals $(\mathrm{N}=144)$

\begin{tabular}{|c|c|c|c|c|}
\hline Abortion/Birth Intervals & $\begin{array}{c}\text { Minimum } \\
\text { (hours) } \\
\end{array}$ & $\begin{array}{c}\text { Maximum } \\
\text { (hours) } \\
\end{array}$ & $\begin{array}{c}\text { Mean } \pm \text { SD } \\
\text { (hours) }\end{array}$ & $\begin{array}{l}\text { Median } \\
\text { (hours) }\end{array}$ \\
\hline $\begin{array}{l}\text { Multiparous (without history of } \\
\text { cesarean section } n=60 \text { ) }\end{array}$ & 2 & 94 & $23.6 \pm 22.5$ & 14.5 \\
\hline Nulliparous $(n=40)$ & 7 & 126 & $32.9 \pm 24.2$ & 27 \\
\hline $\begin{array}{l}\text { Those with histories of caesarian } \\
\text { sections }(n=44)\end{array}$ & 5 & 106 & $32.0 \pm 30.0$ & 18 \\
\hline
\end{tabular}

Feticide was achieved in $99(68.8 \%)$ of the 144 women before the administration of misoprostol. In $71.5 \%$ $(n=103)$, abortion/birth occurred in 48 hours.

In $41(28.5 \%)$ women, misoprostol failed. Misoprostol+oxytocin was administered to $24(16.3 \%)$ of the 41 unsuccessful cases; misoprostol+Foley+oxytocin were administered to $12(8.3 \%)$ of the 41 unsuccessful cases, hysterotomy was performed for the 5 remaining patients whose labor action was unsuccessful.

Feticide was not performed to the remaining 45 cases. The termination durations of the women with and without feticide were examined. The efficacy of the misoprostol protocol was evaluated and compared between the two groups. There was no statistically significant difference in terms of termination durations between the groups $(\mathrm{p}>0.05)$ (Table 4).

Table 4: The evaluation of the gestational weeks and termination durations, and termination condition of women who did and did not undergo feticide

\begin{tabular}{lccc}
\hline The evaluation of cases & $\begin{array}{c}\text { Feticide Performed } \\
(\mathbf{n = 9 9 )}\end{array}$ & $\begin{array}{c}\text { Feticide Not performed } \\
\mathbf{( n = 4 5 )}\end{array}$ & $\mathbf{p}$ \\
\hline \hline Termination status & $70(70.7)$ & $33(73.3)$ & $0.861^{*}$ \\
$\quad$ Successful $\mathbf{~ ( \% ) ~}$ & $29(29.3)$ & $12(26.7)$ & NS** \\
$\quad$ Unsuccessful n (\%) & $21.24 \pm 3.63$ & $21.31 \pm 3.43$ & NS*** \\
Gestational Week (Mean \pm SD) & $35.37 \pm 27.55$ & $32.42 \pm 27.04$ & \\
Termination duration; hours (median) & & & \\
\hline
\end{tabular}

*Fisher's exact test**Student's t-test $* * *$ Mann-Whitney U test NS: Not Significant

Although there were no statistically significant differences between the termination durations and femur length (FL) and abdominal circumference (AC) measurements $(\mathrm{p}>0.05)$, a statistically significant inverse relationship was detected with bi-parietal diameter (BPD) (termina- tion duration is found to shorten with BPD increase) at a level of $34.3 \%$. A statistically significant inverse relationship was detected between termination duration and head circumference $(\mathrm{HC})$ (termination duration is found to shorten with HC increase) at a level of $36.3 \%$ (Table 5, Figure 1, Figure 2). 
Table 5: The relationships of BPD, HC, AC, and FL to termination durations

\begin{tabular}{lll}
\hline & \multicolumn{2}{c}{ Termination Durations } \\
\cline { 2 - 3 } & $\mathbf{r}$ & $\boldsymbol{p}$ \\
\hline \hline FL & 0.150 & 0.192 \\
BPD & -0.343 & $0.020^{*}$ \\
HC & -0.363 & $0.015^{*}$ \\
AC & -0.256 & 0.066 \\
\hline
\end{tabular}

r: Pearson's correlation coefficient

BPD: Biparietal diamater, AC: Abdominal circumference, FL: Femur length, HC: Head circumference

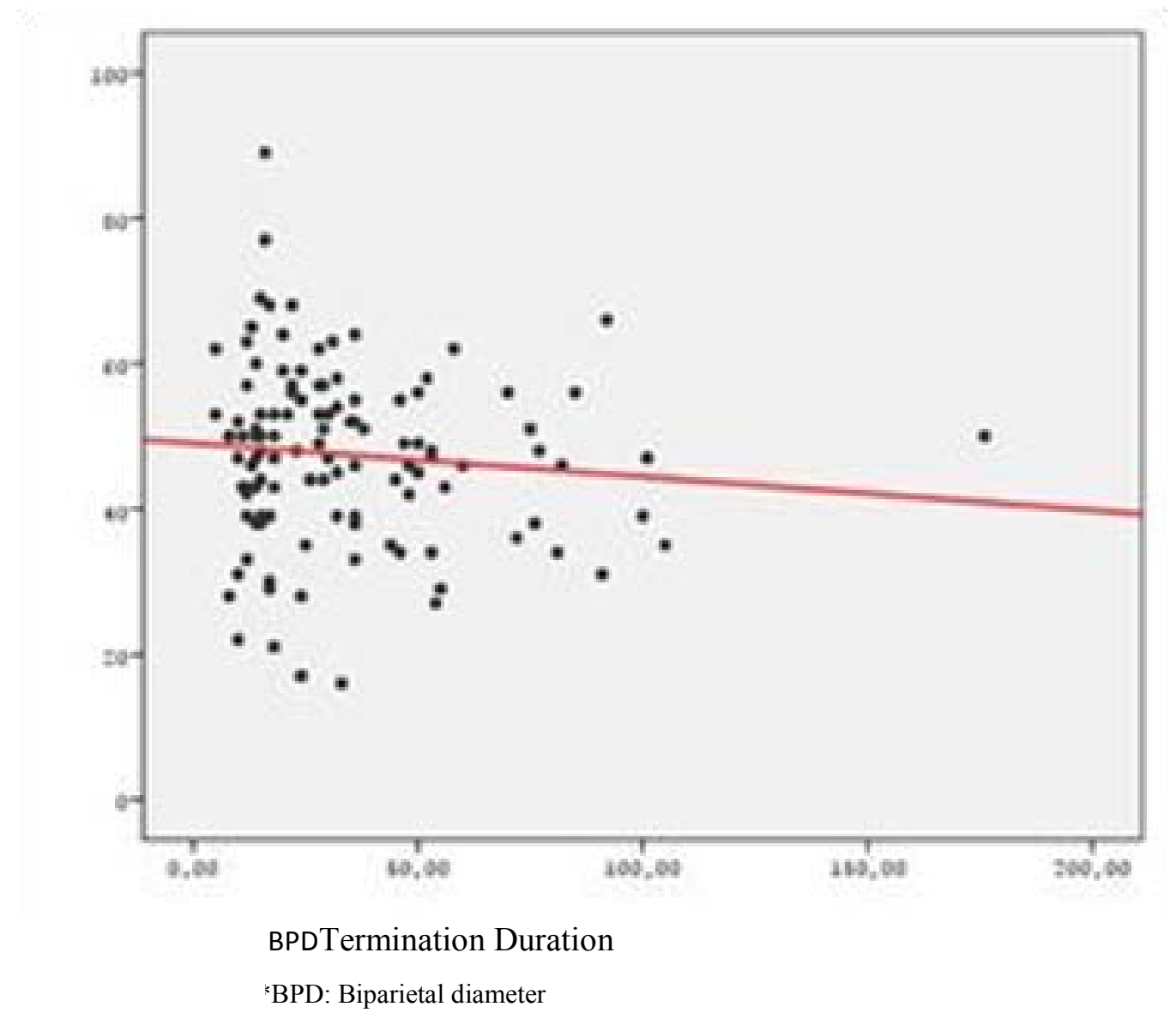

Figure 2. The relationship between termination duration and BDP 
30 of the 44 patients with previous cesarean section resulted in abortion and the success rate was found as $68.9 \%$. In terms of complications, $7(4.8 \%)$ patients had nausea, $5(3.45 \%)$ had vomiting, $6(4.1 \%)$ had diarrhea, 20 $(13.8 \%)$ had fever, $9(6.25 \%)$ had headache and dizziness, and failure of method was determined in 41 (28.4\%) patients. $90(62.5 \%)$ of the remaining 144 women had incomplete abortions and required curettage. In our study, the high rate of curettage after abortion was due to strict control measures for bleeding after abortion and routine cavity control for retained placenta.

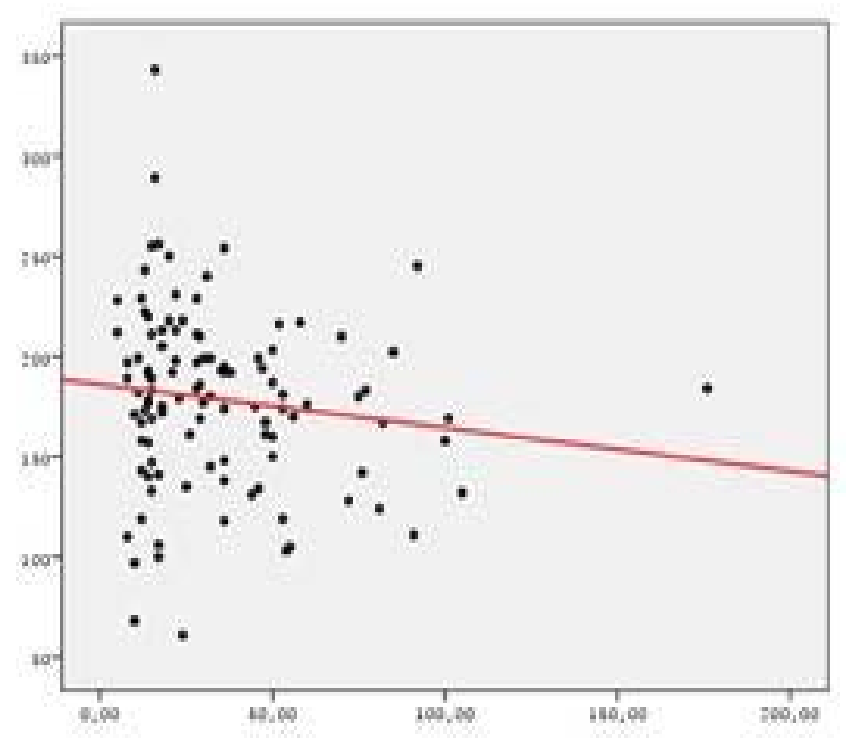

HC Termination Duration

*HC: Head circumference

\section{Figure 3. The relationship between termination duration and HC}

\section{Discussion}

In our retrospectively conducted study, we evaluated the diagnostic and treatment methods as well as the resulting complications of women who were diagnosed as having fetal anomalies; therefore, determining the dispersion of these women according to anomaly type. Abortion/birth was achieved in $71.5 \%$ (103 cases) of women during the first 48 hours. There was no significant difference between the termination durations of the misoprostol protocol among women in whom feticide was and was not performed. There was no significant difference between the termination durations and fetal biometric measurements, except for head diameters.

Gedikbaş1 et al. ${ }^{9}$ evaluated 677 patients and divided these patients into two groups: early termination ( $<23$ weeks) and late termination ( $>23$ weeks). The most common indications in their study were central nervous system anomalies (57.7\%), chromosome anomalies (11.7\%), urogenital anomalies $(8.4 \%)$, cardiovascular system anomalies $(4 \%)$, hydrops $(3.8 \%)$, and maternal infections and diseases $(3.2 \%)$. In multiple pregnancies, high incidences of fetal structural and chromosomal anomalies were detected, along with poor maternal and fetal outcomes. Guillem et al. ${ }^{10}$ scanned a total of 996 patients between 1989 and 2000 and detected fetal structural anomalies in $39 \%$ and chromosome anomalies in 35\%. Different from these studies, central nervous system anomalies were detected as $25 \%$, cardiovascular system anomalies as $20.2 \%$, and chromosomal anomalies as $9.18 \%$ in our study. The number of patients who met the inclusion criteria of our study was smaller.

In our study, abortion/birth occurred in 48 hours with oral-vaginal misoprostol in $71.5 \%(n=103)$ of the women. The method was deemed unsuccessful if abortion/ 
birth did not occur in 48 hours and abortion was achieved with additional methods. In $41(28.5 \%)$ women, misoprostol failed. Misoprostol+oxytocin was administered to $24(16.3 \%)$ of the 41 unsuccessful cases; misoprostol+Foley+oxytocin was administered to $12(8.3 \%)$ of the 41 unsuccessful cases, hysterotomy was performed for the 5 remaining patients whose labor action was unsuccessful. Öztürka et $\mathrm{al}^{11}$ administered $100 \mathrm{mcg}$ misoprostol (Cytotec $200 \mathrm{mcg} / 2$ ) into the posterior fornix of the vagina followed by $100 \mathrm{mcg}$ misoprostol (Cytotec $200 \mathrm{mcg} / 2$ ) every two hours to the 30 cases in the oral group in their study that compared sublingual, vaginal, and oral uses of misoprostol. The same doses were repeated to women who did not have their abortions in 24 hours and their conditions at the end of 48 hours were evaluated. The success rates in the first 24 and 48 hours were $83.3 \%$ $(n=25)$ and $90 \%(n=27)$, respectively. The success rate in the first 48 hours in that study was higher compared with our study. Different than this study, patients with Bishop scores of 3 and below who had no active labor action were included in our study, and the number of patients in their study was smaller compared with our study.

History of cesarean section operations has become common among patients for whom second trimester termination is planned due to increasing rates of cesarean section in today's practice. In women who have had previous cesarean section, uterine ruptures caused by misoprostol cause concerns regarding the use of this $\mathrm{drug}^{12}$. Despite the increasing number of publications reporting that misoprostol can be safely used in patients with transverse incisions in the lower segment of the uterus for termination of pregnancies in the second trimester, the real incidence of uterine rupture risk remains unknown ${ }^{13}$. There are studies reporting that misoprostol use is not contraindicated in patients whose histories are positive for cesarian section operations, but also warning that caution must be taken in their follow-ups due to the high risk of uterine rupture ${ }^{12-14}$. In addition, the safety of misoprostol use for termination of second trimester pregnancies in women with uterine scars is still controversial. Many women with uterine ruptures that occur during the second trimester have a history of previous uterine surgeries $^{15}$. In a study on 720 patients between the gestational weeks of 14-28 who had terminations in the second trimester, 78 had one, 19 had two, and 4 had three previous cesarean section operation histories; Dickinson reported no rupture cases and also that misoprostol was safe to use in patients with histories of cesarian section operations ${ }^{16}$. In their retrospective analysis of 91 patients, Aslan et al. ${ }^{17}$ proved that misoprostol induction doubled the risk of uterine ruptures in patients with histories of cesarian section operations and urged caution in terms of maternal safety. In our study, 44 women had uterine scars due to previous cesarean sections. The minimum and maximum doses of misoprostol administered in this group were 3 and 9 doses respectively, with a mean of $5 \pm 3$ doses. The minimum and maximum times until the abortion were 5 hours and 106 hours, respectively, with a mean of $32 \pm 30$ hours. The success rate in the first 48 hours was $68 \%$ $(n=30)$. Although the number of women with histories of cesarean section operations was low, we detected no complications secondary to the use of misoprostol.

Placenta retention or incomplete abortions are important problems that may arise after terminations in the second trimesters and require surgical intervention. In the first studies regarding misoprostol use for this purpose, more than $80 \%$ of patients were reported to require curettage ${ }^{18,19}$. In our study, $90(62.5 \%)$ of the remaining 144 women had incomplete abortions and required curettage. In Ozturka et al.s' study regarding the comparison of sublingual, vaginal, and oral misoprostol use for second trimester terminations, the incomplete abortion rate was found to be high and similar in all three groups (sublingual $70 \%$, vaginal $69 \%$, and oral $85.2 \%)^{11}$. Bugallo et al..$^{20}$ reported $89 \%$ complete abortion rates in patients until their $22^{\text {nd }}$ gestational weeks.

There was no difference between the women who did and did not undergo feticide in terms of durations of termination or complications in our study. Srisomboon et al. compared the abortion success rates of 89 women with dead and alive fetuses following intravaginal misoprostol administrations at the $12^{\text {th }}, 24^{\text {th }}$, and $48^{\text {th }}$ hours, and reported the abortion rates as $50 \%, 83 \%$ and $97 \%$ and $15 \%, 54 \%$ and $92.5 \%$ respectively. The abortion success rate of intravaginal misoprostol at the $12^{\text {th }}$ and $24^{\text {th }}$ hours was significantly lower in alive fetuses as compared with dead fetuses, but the success rates at the $48^{\text {th }}$ hour were the same. There was no significant difference between the two groups in terms of complications at the $12^{\text {th }}, 24^{\text {th }}$, and 
$48^{\text {th }}$ hours $^{21}$. In one study, the effect of feticide on the duration of labor induction was examined retrospectively and there was no significant difference noticed in the outcome except that the group with feticide tended to have more procedures in the form of manual extraction of placenta or uterine curettage or both $(82.9$ vs. $65.6 \%, \mathrm{P}=$ $0.01)^{22}$. The results also showed that performing feticide procedures had no effect on the termination duration. Another important point of our study is the comparison of BPD, AC, HC, and FL measurements as factors affecting termination duration and the conclusion that termination duration shortens as BPD and $\mathrm{HC}$ increases. However, AC and FL were not observed to have a direct effect on termination duration.

\section{Conclusion}

The use of misoprostol had no effect on termination duration in patients who did and did not undergo feticide procedures in second trimester pregnancy terminations. The administration of misoprostol treatment protocol is effective, safe, and successful in pregnant women with fetal anomalies incompatible with life. Misoprostol decreases the abortion time, and reduces maternal mortality and morbidity.

\section{Acknowledgments}

None.

\section{Consent}

Written informed consent was obtained from all participants. Written consent was obtained from the local Ethics Committee.

\section{Conflict of interest}

The authors declare that they have no conflict of interest. We certify that we had no relationship with companies that may have a financial interest.

\section{References}

1. J Bourke, C Bower, E Blair, A Charles, and M. Knuiman, "The effect of terminations of pregnancy for fetal abnormalities on trends in mortality to one year of age in Western Australia," Paediatric and Perinatal Epidemiology, vol. 19, no. 4, pp. 284-293, 2005.

2. E Z Zimmer, Z Avraham, P Sujoy, I Goldstein, M Bronshtein, "The influence of prenatal ultrasound on the prevalence of congenital anomalies at birth," Prenatal Diagnosis, vol. 17, no. 7, pp. 623-628, 1997.

3. P Guillem, B Fabre, C Cans, E Robert-Gnansia, P S Jouk, "Trends in elective terminations of pregnancy between 1989 and 2000 in a French county (the Isère)," Prenatal Diagnosis, vol. 23, no. 11, pp. 877-883, 2003.

4. C Ramalho, A Matias, O Brandão, N Montenegro, "Critical evaluation of elective termination of pregnancy in a tertiary fetal medicine center during 43 months: correlation of prenatal diagnosis findings and postmortem examination," Prenatal Diagnosis, vol. 26, no. 11, pp. 1084-1088, 2006

5. Babacan A, Yilmaz A, Akpak YK, Mungen E, Gun I, Atay V. Second trimester termination of pregnancy complicated with multiple fetal abnormalities and also placenta percreta. Int J Reprod Contracept Obstet Gynecol. 2014;3:1167-1168.

6. Ercan Ö, Köstü B, Özer A, Serin S, Bakacak M. Misoprostol versus misoprostol and foley catheter combination in $2^{\text {nd }}$ trimester pregnancy terminations. J Matern Fetal Neonatal Med. 2016;29:2810-2812. PubMed.

7. Pongsatha S, Tongsong T. Randomized controlled trial comparing efficacy between a vaginal misoprostol loading and non-loading dose regimen for second-trimester pregnancy termination. J Obstet Gynaecol Res. 2014;40:155160.

8. AI RA, Yapca OE. Vaginal Misoprostol Compared WithBuccal Misoprostol for Termination of Second-Trimester Pregnancy: A Randomized Controlled Trial. Obstet Gynecol. 2015;126:593-598 PubMed .

9. Gedikbaşı A, Gül A, Öztarhan K, et al. Termination of pregnancy and reasons for delayed decisions. J Turkish-German Gynecol Assoc. 2010;11:1-7. PubMed.

10. Guillem P, Fabre B, Cans C, Robert-Gnansia E, Jouk PS. Trends in elective terminations of pregnancy between 1989 and 2000 in a French county (the Isère). Prenat Diagn. 2003;23:877-8 83. PubMed.

11. Özturka Y, Esenler İ. The effectiveness of sublingual, vaginal and oral misoprostol administrations in termination of second trimester pregnancies. Turk J Obstet Gynecol. 2005;2:172-177.

12. Berghahn L, Christensen D, Droste S. Uterine rupture during second trimester abortion associated with misoprostol. Obstet Gynecol. 2001;98:976-977. PubMed.

13. Pongsatha S, Tongsong T. Misoprostol for second trimester termination of pregnancies with prior lowtrans- 
verse cesarean section. Int J Gynecol Obstet. 2003;80:61-62. PubMed.

14. Choy-Hee L, Raynor BD. Misoprostol induction of labor among women with a history of cesarean delivery. Am J Obstet Gynecol. 2001;184:1115-1117. PubMed.

15. Lin CJ, Chien SC, Chen CP. The use of misoprostol in termination of second trimester pregnancy. Taiwan J Obstet Gynecol. 2011;50:275-282.

16. Dickinson JE. Misoprostol for second trimester pregnancy termination in women with a prior cesarean delivery. Obstet Gynecol. 2005;105:352-356. PubMed .

17. Aslan H, Unlu E, Agar M, Ceylan Y. Uterine rupture associated with misoprostol laborinduction in women with previous cesarean delivery. EurJ Obstet Gynecol Reprod Biol. 2004;113:45-48.

18. Thong KJ,Robertson J, Baird dt. Retrospective study of 932 second trimester terminations using gamprost. Prostoglandins. 1992;44:65-74. PubMed.

19. Rodger MW, Baird DT. Pretreatment with mifepristone reduces interval between prostaglandin administration and expulsion in second trimester abortion. $\mathrm{Br} J$ Obstet Gynaecol. 1990;97:41-45. PubMed.

20. Bugallo A, Bigue C, Almeida L. Pregnacy interruption by vaginal misoprostol. Contraseption. 1993; 36:226-229. PubMed.

21. Srisomboon J, Pongpisuttinun S. Efficacy of intracervicovaginal misoprostol in second-trimester pregnancy termination: a comparison betweenlive and dead fetuses. J Obstet Gynaecol Res. 1998;24:1-5.

22. L V Silva, J G Cecatti, J L Pinto E Silva, E Amaral, R Barini, "Feticide does not modify duration of labor induction in cases of medical termination of pregnancy," Fetal Diagnosis and Therapy, vol. 23, no. 3, pp. 192-197, 2008. 